\title{
Loss of Osseointegration
}

National Cancer Institute

\section{Source}

National Cancer Institute. Loss of Osseointegration. NCI Thesaurus. Code C63053.

Problem associated with weakened integration of the device at the bone-implant interface due to loss of fibrous and/or bony tissue and leading to compromised anchorage of the device. i.e. 'Loosening/Lysis.' 